\title{
The burden of PCV13 serotypes in hospitalized pneumococcal pneumonia in Spain using a novel urinary antigen detection test. CAPA study
}

\author{
Rosario Menéndez ${ }^{\mathrm{a}, \mathrm{f}, *}$, Pedro Pablo España ${ }^{\mathrm{b}}$, Emilio Pérez-Trallero ${ }^{\mathrm{c}, \mathrm{f}}$, Ane Uranga ${ }^{\mathrm{b}}$, Raul Méndez ${ }^{\mathrm{a}}$, \\ Catia Cilloniz $^{\mathrm{d}}$, José María Marimón ${ }^{\mathrm{c}, \mathrm{f}}$, Isabel Cifuentes ${ }^{\mathrm{e}}$, Cristina Méndez ${ }^{\mathrm{e}}$, Antoni Torres ${ }^{\mathrm{d}, \mathrm{f}, *}$ \\ ${ }^{a}$ H. Universitario y Politécnico la Fe, Valencia, Spain \\ ${ }^{\mathrm{b}} \mathrm{H}$. Galdakao-Usansolo, Galdácano, Spain \\ ${ }^{c}$ H. Universitario Donostia, San Sebastián, Spain \\ ${ }^{\mathrm{d}}$ H. Clinic, Barcelona, Spain \\ e Pfizer S.L.U., Madrid, Spain \\ ${ }_{\mathrm{f}}^{\mathrm{f}}$ Biomedical Research Center Network for Respiratory Diseases (CIBERES), Madrid, Spain
}

\section{A R T I C L E I N F O}

\section{Article history:}

Received 20 June 2017

Received in revised form 28 July 2017

Accepted 3 August 2017

Available online $\mathrm{xxxx}$

\section{Keywords:}

Pneumococcal pneumonia

PCV13 serotypes

Vaccine

\begin{abstract}
A B S T R A C T
Background: Streptococcus pneumoniae serotypes distribution in community-acquired pneumonia (CAP) requiring hospitalization in adults after introduction of PCV13 in children is not well known. Our aim was to evaluate the distribution of serotypes in pneumococcal pneumonia according to risk factors and comorbidity conditions after the introduction of PCV13 in children in 2010.

Methods: A prospective study from 2011 to 2014 was performed in immunocompetent adults hospitalized with CAP in 3 Spanish hospitals. Microbiological confirmation was obtained using a serotype specific urinary antigen detection test (UAD test), Binax Now and conventional cultures.

Results: 1258 adults were enrolled and pneumococcal pneumonia (invasive disease in $17.7 \%$ ) was confirmed in 368 (29.3\%) and 17.6\% of the any-cause CAP were caused by PVC13 serotypes (3.5\% PCV7 serotypes). Around $60 \%$ of pneumococcal CAP were caused by PCV13 serotypes (74.6\% in invasive episodes vs $57.4 \%$ in non-invasive ones). The most prevalent serotypes in invasive disease were $1,3,7 \mathrm{~F}, 19 \mathrm{~A}$ and 14. No significant differences were observed in the distribution of PCV13 serotypes across the study periods. Regarding comorbidity, the rate of PCV13 serotypes was similar among them, and it was slightly higher in those with no underlying conditions.

Conclusions: Serotypes included in PCV13 caused a significant proportion of CAP in adults with underlying conditions and in healthy adults, with no significant changes in cases due to PCV7 or PCV13 from 2011 to 2014, suggesting an insufficient indirect protection from childhood vaccination. Strategies for implementing pneumococcal vaccination of adults are encouraged to reduce the incidence of pneumococcal episodes.
\end{abstract}

(c) 2017 Elsevier Ltd. All rights reserved.

\section{Introduction}

Pneumococcal disease in adults, including community-acquired pneumonia (CAP) and invasive pneumococcal disease (IPD), is a global health problem, mainly affecting individuals with chronic diseases such as COPD, diabetes mellitus and heart disease. The

* Corresponding authors at: Servicio de Neumología, Hospital Universitario y Politécnico La Fe, Avda, de Fernando Abril Martorell 106, 46026 Valencia, Spain (R. Menéndez). Servicio de Neumología, Hospital Clinic, Barcelona, Spain (A. Torres).

E-mail addresses: rosmenend@gmail.com (R. Menéndez), atorres@clinic.ub.es (A. Torres) increased risk for pneumococcal pneumonia is present all year around [1] and the total disease burden comes mainly from noninvasive episodes, because IPD represents only a fraction [2].

There are more than 90 different pneumococcal serotypes showing diverse clinical expression, invasiveness and outcome. The distribution of circulating pneumococcal serotypes depends on several factors including the presence of underlying diseases, contact with children, and vaccination status, and changes over time making continuous monitoring necessary. Vaccinating children with the 7-valent pneumococcal conjugate vaccine (PCV7) achieved a reduction in adult invasive pneumococcal disease caused by serotypes included in the vaccine and a drift in others 
[3]. The impact that the introduction of the 13-valent pneumococcal conjugate vaccine (PCV13) in 2010 for healthy children has had on the burden of pneumococcal pneumonia in adults due to PCV13 serotypes, particularly CAP without bacteraemia and CAP in high risk groups, is not well known [4]. Moreover, its indirect effect on pneumococcal pneumonia in adults is uncertain, although some impact has been found in the UK, where infant PCV coverage is around 95\% [5]. In adults aged 65 and older, PCV13 has demonstrated efficacy in the prevention of pneumococcal pneumonia and invasive pneumococcal disease [6].

We hypothesized that the distribution of pneumococcal serotypes in adults, in both invasive and non-invasive pneumonia, might have changed after the introduction of PCV13 for children [7] and that this distribution may vary depending on the patients' comorbidity and/or risk factors [8]. Given the fact that hospitalization for pneumonia is not decreasing [9] and that S. pneumoniae is the main causative microorganism, updated information will be a key factor for implementing effective strategies to decrease the incidence of CAP.

The aim of our study was to evaluate the burden of pneumococcal pneumonia in adults with regard to the distribution of PCV13 serotypes using the new UAD test in urine according to comorbidities and/or risk habits in immunocompetent patients after the introduction of PCV13 in children.

\section{Patients and methods}

A prospective multicentre epidemiological study was performed in three tertiary-care teaching hospitals from the National Spanish Health System, covering a population around 900.000 inhabitants (Hospital La Fe 285.000, Hospital Clinic 300.000, Hospital Galdakao 310.000) in immunocompetent adults aged $\geq 18$ years, hospitalized during November 2011 to November 2014. Patients were considered to have CAP when they presented a new radiologic infiltrate accompanied by acute signs and symptoms suggestive of lower respiratory tract infection. Exclusion criteria were previous hospitalization that lasted at least $48 \mathrm{~h}$ within the 2 weeks prior to the current admission, active pulmonary tuberculosis, sickle cell anemia, immunosuppression (HIV infection/AIDS or immune suppression by any other cause), current cancer treatment or cancer treatment during the year prior to the study, and functional or anatomic asplenia. The ethics committee at each hospital approved the study and patients signed written informed consents.

Data on age, gender, smoking, alcohol consumption, prior antimicrobial treatment, comorbidity conditions (chronic obstructive pulmonary disease, asthma, diabetes mellitus, chronic heart failure, chronic kidney failure, chronic liver disease, cerebrovascular disease, cancer), history of previous pneumonia, of previous hospitalization and of flu and pneumococcal vaccination were recorded for each patient. Initial severity was evaluated using the Pneumonia Severity Index (PSI) and the CURB-65 score. In the chest X-ray, we recorded the extension of infiltrate and the presence of pleural effusion.

During hospitalization the appearance of new complications, episodes requiring mechanical ventilation, treatment failure, admission into the intensive care unit (ICU), length of stay, and outcome were recorded.

\subsection{Microbiological results}

Microbiological studies were performed at each participating hospital according to standard clinical practice and based on clinical judgment of the attending physician. Cultures were done on available samples from blood ( $\mathrm{n}$ : 844), pleural fluid (n: 93), or other body fluids, sterile under normal conditions, and/or good quality sputum ( $<10$ epithelial cells and $>25$ leukocytes per field; magnification $x$ 100) (n: 444). Urinary antigen tests performed were Binax Now S. pneumoniae Urinary Antigen Test (n:1190) and Binax Now L. pneumophila Urinary Antigen Test (n:1190). Other tests were performed to 322 patients, like PCR for viruses in 244 patients and serological studies for the detection of antibodies to Chlamydophila pneumoniae, Mycoplasma pneumoniae, Coxiella burnetii and L. pneumophila.

Urine samples obtained were processed and stored between $-20^{\circ} \mathrm{C}$ and $-70^{\circ} \mathrm{C}$ at each participating hospital in all patients (n: 1258) until they were sent to the sponsor's central laboratory in Pearl River, New York, USA to perform the serotype specific urinary antigen detection (UAD) test. This test consists of an immunochromatographic membrane assay using monoclonal antibodies of the serotype-specific capsular polysaccharide. The assay detects the thirteen $S$. pneumoniae serotypes included in PCV13 (serotypes 1, 3, 4, 5, 6A, 6B, 7F, 9V, 14, 18C, 19A, 19F and 23F) and has a sensitivity and specificity of $98 \%$ and $100 \%$, respectively [10].

Pneumococcal isolates were identified by the optochinsusceptibility and bile solubility tests and they were stored frozen in skimmed milk at $-40{ }^{\circ} \mathrm{C}$ or lower until delivery to the central laboratory [Microbiology Department, University Hospital Donostia, Donostia-San Sebastián (Spain)]. Serotyping was performed by multiplex-PCR [11] and confirmed by the Quellung reaction using polyclonal antisera (Statem Serum Institute, Copenhagen, Denmark), antibiotic susceptibility testing (broth microdilution, CLSI guidelines) and genotyping (MLST) were performed by this central laboratory on all isolates sent.

\subsection{Definitions}

Pneumococcal CAP etiology was considered present if any microbiological test was positive (Binax Now test, UAD test, blood culture, pleural culture and sputum culture). Invasive disease was confirmed if $S$. pneumoniae was isolated in blood and/or pleura fluid. Non-invasive disease was defined as confirmed pneumococcal CAP (by UAD test or Binax Now test) for which blood and/or pleura fluid culture results were negative. Pneumococcal serotypes were categorized as follows: $4,6 \mathrm{~B}, 9 \mathrm{~V}, 14,18 \mathrm{C}, 19 \mathrm{~F}$ and $23 \mathrm{~F}$ as "PCV7" serotypes; 1, 3, 5, 6A, 7F, 19A plus PCV7 included serotypes as "PCV13" serotypes.

Complicated CAP at presentation was considered if one of the following was present: multilobar infiltrates, bacteraemia, pleural effusion or empyema, respiratory failure, severe sepsis or septic shock. Severe sepsis was considered present in cases of any organ failure other than respiratory failure. Treatment failure was considered according to Spanish guidelines [12]. Clinical stability with modified Halm criteria [13] was considered achieved when vital signs of a patient were stable for a $24 \mathrm{~h}$ period (i.e. heart rate $<100$ beats per minute; respiratory rate <24 breaths per minute; axillary temperature $<37.2^{\circ} \mathrm{C}$; systolic blood pressure $>90 \mathrm{mmHg}$; oxygen saturation $>90 \%$; good level of consciousness; tolerance to oral via.

\subsection{Statistical analyses}

Data analysis was performed using SPSS 19.0 software and EPIDAT 3.0. For comparisons of independent samples, the Pearson chisquared test (or the exact Fisher test for $2 \times 2$ tables or likelihood ratio for mxn tables, if necessary) for qualitative variables and Student's $t$ test, single factor ANOVA or its non-parametric equivalent U-Mann-Mann, H-Kruskal_Wallis for quantitative variables were used. The assumptions of normality and homoscedasticity of the variables were studied for use of parametric tests. The pneumococcal CAP group was stratified as invasive and non-invasive CAP. 


\section{Results}

A total of 1258 patients were recruited from November 2011 to November 2014 (Table 1). Microbiological aetiology was found in 573 patients (45.5\%): 368 (29.3\%) S. pneumoniae, 24 (1.9\%) L. pneumophila, 19 (1.5\%) Staphylococcus aureus, 21 (1.7\%) Influenza virus, $12(1.0 \%)$ other virus, and 129 (10.3\%) others microorganisms (Fig. 1). Pneumococcal CAP was diagnosed in 368 patients. No significant changes were observed in the percentage of all-cause CAP due to $S$. pneumoniae during the three years of the study (Table 2). Of the 368 cases of pneumococcal CAP, 65 (17.7\%) were invasive and $303(82.3 \%)$ were non-invasive. Pneumococcal CAP cases showed a higher proportion of asthmatic patients (11.4\% vs $7.8 \%$; $p<0.05$ ) and alcohol abuse (4.9\% vs $2.4 \% ; p<0.05$ ), higher PSI (45.8\% vs 39\% for PSI risk class IV-V; $p<0.05$ ) and CURB65 (1.49 vs $1.26 ; p<0.001)$ punctuations and more ICU admission $(13.4 \%$ vs $9.4 \% ; p<0.05)$.

\subsection{Serotype distribution}

Serotype distribution showed that $17.6 \%$ of all-cause CAP was caused by serotypes included in PCV13 without significant differences throughout the study period. Pneumococcal pneumonia was caused in $60 \%$ of the cases by PCV13 serotypes and it was greater in invasive diseases (74.6\%) with no significant differences during the three years of the study (Table 2). The percentage of cases due to PCV7 serotypes was $12 \%$ for pneumococcal pneumonia and 9.5\% in invasive episodes. The distribution of PCV13 serotypes identified in invasive pneumococcal pneumonia and in non-invasive pneumonia is shown in Fig. 2. Although invasive cases were caused more frequently by PCV13 serotypes than were in non-invasive cases (74.6\% vs $57.4 \% ; p<0.05$ ), no significant differences were observed for individual serotypes except for sero-

Table 1

Characteristics of study population in the whole cohort and in pneumococcal CAP.

\begin{tabular}{|c|c|c|}
\hline & $\begin{array}{l}\text { Any-cause CAP } \\
(n=1258) \\
N(\%)\end{array}$ & $\begin{array}{l}\text { Pneumococcal CAP } \\
(n=368) \\
N(\%)\end{array}$ \\
\hline \multicolumn{3}{|l|}{ Demographic data } \\
\hline Age [mean(SD)] & $67.41(17.90)$ & $67.64(17.82)$ \\
\hline Gender (male) & $763(60.7 \%)$ & $216(58.7 \%)$ \\
\hline Spanish nationality & $1211(96.3 \%)$ & $352(95.7 \%)$ \\
\hline \multicolumn{3}{|l|}{ Underlying conditions $(\geq 1)$} \\
\hline No underlying condition & $155(12.3 \%)$ & $54(14.7 \%)$ \\
\hline COPD & $232(18.4 \%)$ & $73(19.8 \%)$ \\
\hline Chronic heart failure & $159(12.6 \%)$ & $37(10.1 \%)$ \\
\hline Diabetes mellitus & 301 (23.9\%) & 77 (20.9\%) \\
\hline Chronic liver disease & $42(3.3 \%)$ & $11(3.0 \%)$ \\
\hline Chronic renal failure & $117(9.3 \%)$ & $33(9.0 \%)$ \\
\hline Stroke & $101(8.0 \%)$ & $27(7.3 \%)$ \\
\hline Asthma & $111(8.8 \%)$ & $42(11.4 \%)$ \\
\hline Cured neoplasia & $112(8.9 \%)$ & $37(10.1 \%)$ \\
\hline Previous hospitalization & $27(2.1 \%)$ & $8(2.2 \%)$ \\
\hline Previous pneumonia & $206(16.4 \%)$ & $63(17.1 \%)$ \\
\hline \multicolumn{3}{|l|}{ Toxic habits } \\
\hline Smoking ${ }^{\mathrm{a}}$ & $201(16.0 \%)$ & $59(16.0 \%)$ \\
\hline Alcoholism ${ }^{\mathrm{b}}$ & 39 (3.1\%) & $18(4.9 \%)$ \\
\hline \multicolumn{3}{|l|}{ Vaccination history } \\
\hline Flu vaccine & $587(46.7 \%)$ & $161(43.8 \%)$ \\
\hline Pneumococcal vaccine & 99 (7.9\%) & $22(6.0 \%)$ \\
\hline \multicolumn{3}{|l|}{ PSI score } \\
\hline I-II & $403(32.2 \%)$ & $120(32.7 \%)$ \\
\hline III & $336(26.8 \%)$ & $79(21.5 \%)$ \\
\hline IV & $421(33.6 \%)$ & $131(35.7 \%)$ \\
\hline V & $93(7.4 \%)$ & 37 (10.1\%) \\
\hline
\end{tabular}

a Smoking: smokers of $\geq 10$ cigarettes per day within the previous year or quitting smoking less than 6 months before.

b Alcoholism: intake $\geq 80$ gr per day during at least the previous year. type 1 ( $27 \%$ vs $4.6 \%$; $p<0.001)$. The most prevalent serotypes (representing at least 5\%) identified in the invasive cases were 1 (27\%), 3 (16\%), 7F (14.3\%), 14 (7.9\%), 19A (7.9\%) and 8 (6.3\%). For the non-invasive pneumococcal pneumonia cases, the most frequent serotypes were 3 (23.1\%), 7F (6.9\%) 19A (6.9\%) and 14 (5.3\%). No significant differences were found within each hospital for all-cause CAP or pneumococcal pneumonia cases due to PCV7 or PCV13 serotypes during the study period.

The distribution of PCV13 serotypes according to initial presentation of pneumococcal pneumonia cases is depicted in Supplemental file 1 . Serotype 3 was the most frequent serotype in all the variables related to initial severity except for cases complicated with bacteraemia which was serotype 1 , with empyema which were serotypes 3 and 1 and, for cases complicated with severe sepsis which was serotype 7F.

Regarding serotype distribution according to underlying conditions, serotypes included in PCV13 accounted for $72.7 \%$ of cases in patients with no underlying conditions, for 69.3\% (70/101) in patients with 1 underlying condition, and for 52.1\% (110/211) in patients with at least two underlying conditions. Table 3 shows serotypes distribution according to the most prevalent underlying conditions in CAP and pneumococcal CAP episodes. In those with no underlying conditions there was a significantly higher total burden of PCV13 serotypes compared to patients with at least one underlying condition (72.2\% vs $57.7 \%$; $=0.032$ ).

\subsection{Complications, LOS and outcome}

During hospitalization, 358 patients presented new complications. Death occurred in 35 patients (2.8\%), most of them (32 cases) during hospitalization. PCV13 serotypes distribution according to complications, length of stay and mortality are shown in Supplemental file 2. Serotype 3 was the most frequent in patients presenting complications during hospitalization, with the exception of the cases complicated with sepsis where 3 and 14 were equally frequent serotypes. Serotype 3 was also the most frequent serotype in patients requiring invasive mechanical ventilation, in cases with a length of stay at hospital or in the ICU longer than 7 days and, in cases taking more than 4 days to become clinically stable.

\section{Discussion}

The most outstanding findings of our study are 1 . More than $60 \%$ of pneumococcal CAP cases in immunocompetent adults were caused by PCV13 serotypes (74.6\% in invasive episodes and $57.4 \%$ in non-invasive episodes) showing no significant changes from 2011 to 2014. 2. The most frequent serotypes in pneumococcal CAP were 3, 1, 7F and 19A. 3. The percentage of CAP caused by PCV13 serotypes varied slightly according to the presence of underlying conditions (from $49.2 \%$ in previous CAP to $64.9 \%$ in diabetics) and was higher in patients without comorbidities and/or risk factors.

In our cohort, $29.3 \%$ of patients had pneumococcal CAP of whom $17.7 \%$ had invasive episodes. Regarding underlying conditions, $85.3 \%$ of our patients with pneumococcal CAP had at least one comorbidity and/or risk habit. Some chronic conditions, such as respiratory disease, current smoking, heart disease or diabetes, increase the risk for pneumococcal pneumonia between 3 and 9fold [1]. That increase in risk is accumulative in patients with multiple risk factors mainly in those with 3 or more chronic conditions [14,15].

Regarding circulating serotypes, there is much more information in IPD [16-18] than on non-invasive disease despite the fact that pneumococcal CAP is responsible for the major burden of pneumococcal disease. Prior to PCV13 introduction, data on secular 


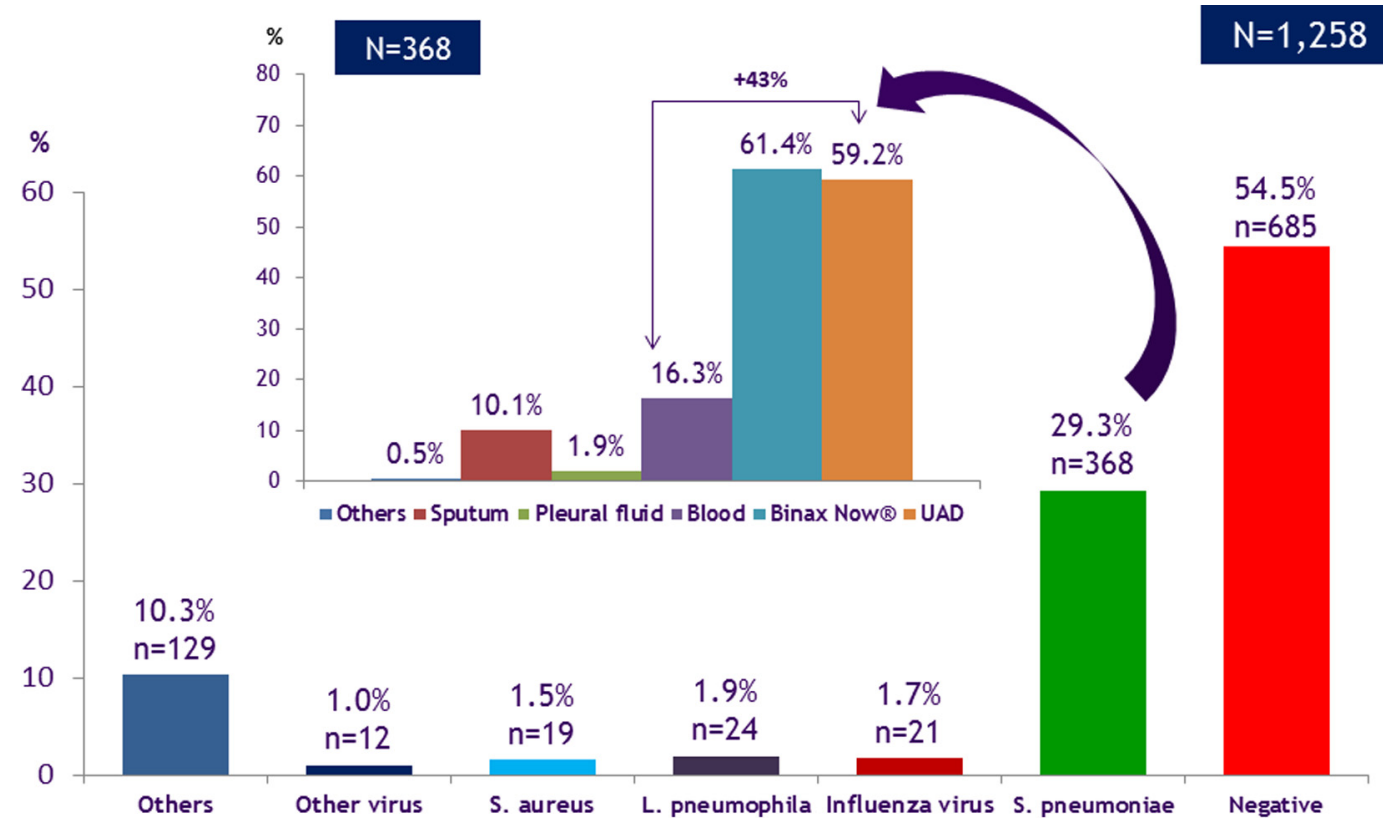

Fig. 1. Microbiological results of CAP $(N=1258)$ and pneumococcal CAP $(N=368)$. 2011-2014.

Table 2

Distribution of PCV7 and PCV13 vaccine serotypes in any-cause CAP and in invasive ${ }^{\mathrm{a}}$ and non-invasive ${ }^{\mathrm{b}}$ pneumococcal CAP by study period.

\begin{tabular}{|c|c|c|c|c|c|}
\hline & Nov 2011-Nov 2012 & Nov 2012-Nov 2013 & Nov 2013 -Nov 2014 & Total & $p$-value \\
\hline Disease & $\mathrm{N}(\%)$ & $\mathrm{N}(\%)$ & N (\%) & $\mathrm{N}(\%)$ & \\
\hline Any-cause CAP & $N=431$ & $N=434$ & $N=393$ & $N=1258$ & \\
\hline PCV13 serotypes & $77(17.9)$ & $85(19.6)$ & $59(15.0)$ & $221(17.6)$ & 0.217 \\
\hline PCV7 serotypes & $13(3.0)$ & $18(4.1)$ & $13(3.3)$ & $44(3.5)$ & 0.649 \\
\hline \multirow[t]{4}{*}{ Most prevalent serotypes } & $3(25 ; 5.8 \%)$ & $3(32 ; 7.4 \%)$ & $3(23 ; 5.9 \%)$ & $3(80 ; 6.4 \%)$ & 0.571 \\
\hline & $1(13 ; 3.0 \%)$ & $1(12 ; 2.8 \%)$ & $1(6 ; 1.5 \%)$ & $1(31 ; 2.5 \%)$ & 0.312 \\
\hline & $7 \mathrm{~F}(11 ; 2.6 \%)$ & $7 \mathrm{~F}(10 ; 2.3 \%)$ & $7 F(9 ; 2.3 \%)$ & $7 F(30 ; 2.4 \%)$ & 0.962 \\
\hline & $19 \mathrm{~A}(8 ; 1.9 \%)$ & $19 \mathrm{~A}(12 ; 2.8 \%)$ & $19 A(6 ; 1.5 \%)$ & $19 \mathrm{~A}(26 ; 2.1 \%)$ & 0.435 \\
\hline CAP due to $S$. pneumoniae & $114(26.5)$ & $143(32.9)$ & $111(28.2)$ & $368(29.3)$ & 0.097 \\
\hline PCV13 serotypes & $77(67.5)$ & $85(59.4)$ & $59(53.2)$ & $221(60.1)$ & 0.085 \\
\hline PCV7 serotypes & $13(11.4)$ & $18(12.6)$ & $13(11.7)$ & $44(12.0)$ & 0.954 \\
\hline Invasive $\mathrm{CAP}^{\mathrm{a}}$ & $19(16.7)$ & $31(21.7)$ & $15(13.5)$ & $65(17.7)$ & 0.225 \\
\hline PCV13 serotypes & $13(72.2)$ & $25(83.3)$ & $9(60.0)$ & $47(74.6)$ & 0.309 \\
\hline PCV7 serotypes & $0(0.0)$ & $6(20.0)$ & $0(0.0)$ & $6(9.5)$ & 0.008 \\
\hline Non-PCV13 serotypes & $5(26.3)$ & $5(16.1)$ & $6(40)$ & $16(24.6)$ & 0.309 \\
\hline \multirow[t]{13}{*}{ Most prevalent serotypes } & $1(6 ; 31.5 \%)$ & $1(8 ; 25.8 \%)$ & $1(3 ; 20 \%)$ & $1(17 ; 26.1)$ & 0.688 \\
\hline & $3(2 ; 10.5 \%)$ & $3(5 ; 16.1 \%)$ & $3(3 ; 20 \%)$ & $3(10 ; 15.4 \%)$ & 0.768 \\
\hline & $7 F(4 ; 21 \%)$ & $7 F(3 ; 9.8 \%)$ & $7 \mathrm{~F}(2 ; 13.4 \%)$ & $7 F(9 ; 13.8 \%)$ & 0.517 \\
\hline & $14(0)$ & $14(5 ; 16.1 \%)$ & $14(0)$ & $14(5 ; 7.7 \%)$ & 0.019 \\
\hline & $19 A(1 ; 5.3 \%)$ & $19 A(3 ; 9.7 \%)$ & $19 A(1 ; 6.7 \%)$ & $19 A(5 ; 7.7 \%)$ & 0.839 \\
\hline & $8(1 ; 5.3 \%)$ & $8(2 ; 6.4 \%)$ & $8(1 ; 6.7 \%)$ & $8(4 ; 6.1 \%)$ & 0.986 \\
\hline & $22 F(1 ; 5.3 \%)$ & $22 F(1 ; 3.2 \%)$ & $22 F(1 ; 6.7 \%)$ & $22 F(3 ; 4.6 \%)$ & 0.869 \\
\hline & $6 C(2 ; 10.5 \%)$ & $6 C(0)$ & $6 C(0)$ & $6 C(2 ; 3.0 \%)$ & 0.075 \\
\hline & $11 A(0)$ & $11 A(1 ; 3.2 \%)$ & $11 A(1 ; 6.7 \%)$ & $11 A(2 ; 3.0 \%)$ & 0.445 \\
\hline & $12 F(0)$ & $12 F(0)$ & $12 F(2 ; 13.4 \%)$ & $12 F(2 ; 3.0 \%)$ & 0.051 \\
\hline & $13(1 ; 5.3 \%)$ & $13(1 ; 3.2 \%)$ & $13(0)$ & $13(2 ; 3.0 \%)$ & 0.537 \\
\hline & $29(0)$ & $29(0)$ & $29(1 ; 6.7 \%)$ & $29(1 ; 1.5 \%)$ & 0.232 \\
\hline & $9 \mathrm{~V}(0)$ & $9 \mathrm{~V}(1 ; 3.2 \%)$ & $9 \mathrm{~V}(0)$ & $9 \mathrm{~V}(1 ; 1.5)$ & - \\
\hline Non-invasive $\mathrm{CAP}^{\mathrm{b}}$ & $95(83.3)$ & $112(78.3)$ & $96(86.5)$ & $303(82.3)$ & 0.225 \\
\hline PCV13 serotypes & $64(67.4)$ & $60(53.6)$ & $50(52.1)$ & $174(57.4)$ & 0.057 \\
\hline PCV7 serotypes & $13(13.7)$ & $12(10.7)$ & $13(13.5)$ & $38(12.5)$ & 0.759 \\
\hline \multirow[t]{6}{*}{ Most prevalent serotypes } & $3(23 ; 24.2 \%)$ & $3(27 ; 24.1 \%)$ & $3(20 ; 20.8 \%)$ & $3(70 ; 23.1 \%)$ & 0.813 \\
\hline & $7 F(7 ; 7.4 \%)$ & $7 F(7 ; 6.2 \%)$ & 7F (7; 7.3\%) & $7 F(21 ; 6.9 \%)$ & 0.937 \\
\hline & $19 A(7 ; 7.4 \%)$ & $19 A(9 ; 8.0 \%)$ & $19 A(5 ; 5.2 \%)$ & $19 \mathrm{~A}(21 ; 6.9 \%)$ & 0.701 \\
\hline & $14(6 ; 6.3 \%)$ & $14(5 ; 4.5 \%)$ & $14(5 ; 5.2 \%)$ & $14(16 ; 5.3 \%)$ & 0.839 \\
\hline & $1(7 ; 7.4 \%)$ & $1(4 ; 3.6 \%)$ & $1(3 ; 3.1 \%)$ & $1(14 ; 4.6 \%)$ & 0.327 \\
\hline & $6 \mathrm{~A}(6 ; 6.3 \%)$ & $6 \mathrm{~A}(1 ; 0.9 \%)$ & $6 \mathrm{~A}(2 ; 2.0 \%)$ & $6 \mathrm{~A}(9 ; 2.9 \%)$ & 0.067 \\
\hline
\end{tabular}

\footnotetext{
a Isolate of $S$. pneumoniae in blood and/or pleural fluid. Among 65 cases identified, 2 isolates not serotyped.

b Confirmed pneumococcal CAP (by UAD or Binax Now) for which blood and/or pleural fluid culture result were negative. Data of non-PCV13 serotypes causing invasive CAP are included in italic font.
} 


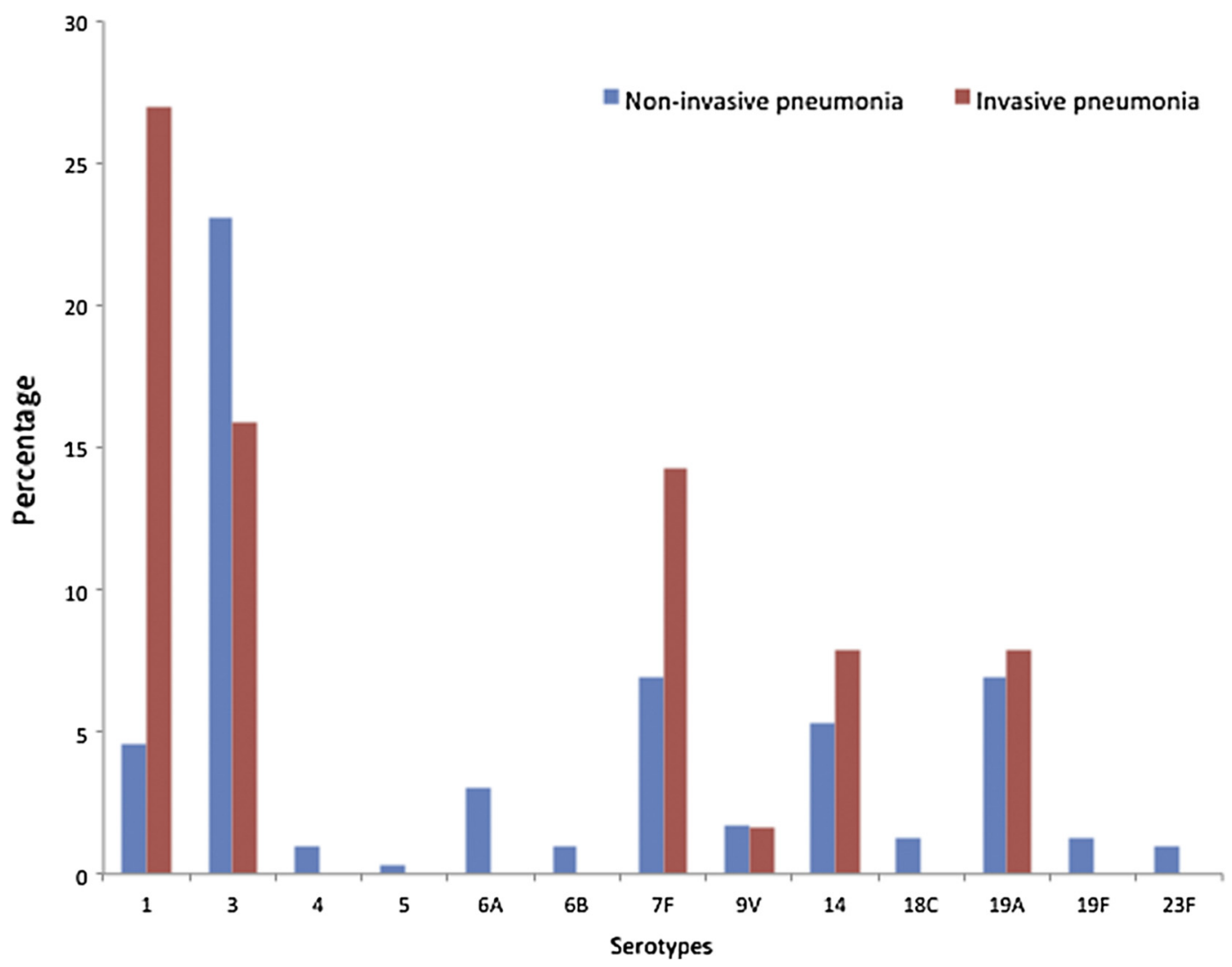

Fig. 2. Distribution of PCV13 serotypes in pneumococcal pneumonia $(n=368)$ cases according to the presence of invasive disease.

Table 3

Serotype distribution according to presence of underlying conditions. ${ }^{a}$

\begin{tabular}{|c|c|c|c|c|c|c|c|c|}
\hline & $\begin{array}{l}\text { Diabetes } \\
\text { mellitus } \\
\mathrm{n}(\%)\end{array}$ & $\begin{array}{l}\text { COPD } \\
\mathrm{n}(\%)\end{array}$ & $\begin{array}{l}\text { Previous } \\
\text { CAP } \\
\mathrm{n}(\%)\end{array}$ & $\begin{array}{l}\text { Smoking } \\
\mathrm{n}(\%)\end{array}$ & $\begin{array}{l}\text { Heart } \\
\text { failure } \\
\mathrm{n}(\%)\end{array}$ & $\begin{array}{l}\text { Chronic renal } \\
\text { failure } \\
\mathrm{n}(\%)\end{array}$ & $\begin{array}{l}\text { Asthma } \\
\mathrm{n}(\%)\end{array}$ & $\begin{array}{l}\text { No underlying } \\
\text { conditions } \\
\mathrm{n}(\%)\end{array}$ \\
\hline Any- cause CAP. 1258 & 301 (23.9) & $232(18.4)$ & $206(16.4)$ & $201(16.0)$ & 159 (12.6) & $117(9.3)$ & $111(8.8)$ & $155(12.3)$ \\
\hline PCV13 serotypes & $50(16.6)$ & $37(15.9)$ & $31(15.0)$ & $37(18.4)$ & $20(12.6)$ & $20(17.1)$ & $22(19.8)$ & $39(25.2)$ \\
\hline PCV7 serotypes & $6(2.0)$ & $9(3.9)$ & $7(3.4)$ & $5(2.5)$ & $8(5.0)$ & $3(2.6)$ & $3(2.7)$ & $5(3.2)$ \\
\hline Pneumococcal CAP 368 (29.3) & $77(25.6)$ & $73(31.5)$ & $63(30.6)$ & $59(29.4)$ & $37(23.3)$ & $33(28.2)$ & $42(37.8)$ & $54(34.8)$ \\
\hline PCV13 serotypes & $50(64.9)$ & $37(50.7)$ & $31(49.2)$ & $37(62.7)$ & $20(54.1)$ & $20(60.6)$ & $22(52.4)$ & $39(72.2)$ \\
\hline PCV7 serotypes & $6(7.8)$ & $9(12.3)$ & $7(11.1)$ & $5(8.5)$ & $8(21.6)$ & $3(9.1)$ & $3(7.1)$ & $5(9.3)$ \\
\hline Invasive $\mathrm{CAP}^{\mathrm{b}}$ & $11(14.3)$ & $4(5.5)$ & $4(6.3)$ & $16(27.1)$ & $6(16.2)$ & $6(18.2)$ & $8(19.0)$ & $12(22.2)$ \\
\hline \multicolumn{9}{|l|}{$65(17.7)$} \\
\hline PCV13 serotypes & $9(81.8)$ & $3(75.0)$ & $3(75.0)$ & $10(62.5)$ & $3(50.0)$ & $5(83.3)$ & $7(87.5)$ & $12(100.0)$ \\
\hline PCV7 serotypes & $0(0.0)$ & $1(25.0)$ & $0(0.0)$ & $0(0.0)$ & $0(0.0)$ & $0(0.0)$ & $1(12.5)$ & $2(16.7)$ \\
\hline Non-invasive $\mathrm{CAP}^{\mathrm{C}}$ & $66(85.7)$ & $69(94.5)$ & $59(93.7)$ & $43(72.9)$ & $31(83.8)$ & $27(81.8)$ & $34(81.0)$ & $42(77.8)$ \\
\hline \multicolumn{9}{|l|}{$303(82.3)$} \\
\hline PCV13 serotypes & $41(62.1)$ & $34(49.3)$ & $28(47.5)$ & $27(62.8)$ & $17(54.8)$ & $15(55.6)$ & $15(44.1)$ & $27(64.3)$ \\
\hline PCV7 serotypes & $6(9.1)$ & $8(11.6)$ & $7(11.9)$ & $5(11.6)$ & $8(25.8)$ & $3(11.1)$ & $2(5.9)$ & $3(7.1)$ \\
\hline
\end{tabular}

a Patients might have more than one underlying condition.

b Isolate of $S$. pneumoniae in blood and/or pleural fluid. Among 65 cases identified, 2 isolates not serotyped.

c Confirmed pneumococcal CAP (by UAD or Binax Now) for which blood and/or pleural fluid culture result were negative.

trend of pneumococci serotypes in Spain indicated that the percentage of PCV13 isolates among IPD cases showed an increasing trend until 2001, reaching nearly $90 \%$, followed by a decrease, which was most prominent since 2010 when they accounted for around $70 \%$ of the isolates. A decrease of $32.3 \%$ was found in the percentage of PCV13 isolates from 2010 to 2013 [19].

We studied the burden of pneumococcal disease from PCV13 serotypes using a serotype specific urinary antigen detection test (Pfizer UAD test) [10] in addition to conventional serotyping of strains from blood or pleural fluid cultures. These tests achieve a more accurate determination of pneumococci serotypes in non- invasive pneumonia [20-22] than can be achieved by serotyping samples from lower respiratory tract. In fact, some serotypes might be underestimated if only invasive disease is taken into account and samples from the lower respiratory tract might confound colonization with infection.

Our findings showed that 13 years after introduction of PCV7/ PCV13 for children in our country, mainly with private funding and with an estimated uptake of around 61\% [23], 17.6\% of CAP was caused by PCV13 serotypes, and that $3.5 \%$ of CAP was caused by PCV7 serotypes, indicating that a significant proportion of adults continue to develop vaccine serotype CAP. In addition, these 
results also suggest that childhood immunization has not eliminated the circulation of PCV7 serotypes. This finding is similar to that of Sherwin et al. [21] in USA and Bewick et al. in UK [20], with a higher infant uptake, pointing out the insufficient indirect protection from pneumococcal CAP in adults as a result of childhood vaccination.

Serotype 3 was the most prevalent serotype in CAP accounting for $21.7 \%$ of the cases, followed by far by serotypes $1,7 \mathrm{~F}$ and $19 \mathrm{~A}$. Nevertheless, serotype 1 was the most prevalent (17 cases; $27 \%$ ) in invasive pneumococcal CAP, whereas serotype 3 was more frequent (70 cases; $23 \%$ ) in non-invasive pneumococcal CAP. Serotypes 1 and 3 have been associated with complicated pneumonia, specifically with empyema and necrotizing pneumonia $[24,25]$. The different distribution of serotypes 1 and 3 in invasive and non-invasive cases found in our study does not seem to be explained by differences in nasopharyngeal carriage and pathogenic potential. Serotype 1 is rarely identified in carriage but has high pathogenic potential, whereas serotype 3 has low carriage prevalence and high pathogenic potential [26]. On the other hand, the high prevalence of PCV13 serotypes on pneumococcal CAP found in our study, mainly for serotypes 3, 1, 7F and 19A, suggests that indirect protection of adults against pneumococcal CAP is not as evident as that observed against IPD $[3,18]$ or against pneumococcal CAP $[5,26]$ in countries with well-established pneumococcal vaccination programs for children using PCV7/PCV13. Nevertheless, we observed a non-significant decreasing trend in the percentage of non-invasive CAP cases due to PCV7/13 serotypes throughout the study period. This decrease could be explained by a possible early indirect effect of PCV13 infant vaccination, as previously reported [3,5]. However it should be taken into account that a longer period of time and higher vaccine uptake is needed for the herd effect to substantially decrease pneumococcal disease at a population level [27].

We confirmed more invasive episodes in patients who smoked (27\%) followed by patients with asthma, and chronic renal and heart disease. Interestingly, there were a considerable number of episodes in patients without underlying conditions (22\%). With regard to the serotypes identified in invasive cases, we found that the percentage of PCV13 serotypes ranged from $100 \%$ in those with no underlying conditions to $75-85 \%$ in those with diabetes, COPD, chronic renal disease and asthma. Ardanuy et al. [17] studied IPD episodes in patients $>65$ years and found that PCV13 serotypes (19A, 3, 7F, 14 and 1) were the most prevalent, causing $59.3 \%$ of episodes.

The distribution of PCV13 serotypes in pneumococcal disease related to comorbidity conditions and/or risk factors is quite unknown although it has been suggested that different conditions may predispose to different serotypes. The highest percentage of PCV13 serotypes was found in diabetics (64.9\%), smokers (62.7\%), and in patients with chronic renal disease, while it was lowest in those with previous pneumonia (49.2\%). Interestingly in those with no underlying conditions the total burden of PCV13 serotypes was the highest, and significantly higher than in patients with at least one underlying condition. PCV7 serotypes showed higher percentages in patients with heart disease or COPD. Except in asthmatic patients, serotype 3 was the most prevalent, followed by $19 \mathrm{~A}$ and $7 F$. In COPD patients with exacerbations and pneumonia, serotypes $10 \mathrm{~A}, 11 \mathrm{~A}$, and $33 \mathrm{~F}$ were found more frequently found in sputum while $1,3,5$ and 8 were more frequent in blood causing bacteraemia [28].

The serotypes most frequently found in invasive episodes were 3, 1, 7F and 19A. Serotype 3 has been frequently associated with complications such as empyema [29], septic shock [30] and respiratory failure [31]. The different capacities of serotypes to adhere to the respiratory epithelium due to the expression of different adhesins has been found to be related to virulence [32]. In an animal model, it has been shown that the different capsular types pro- voke diverse inflammatory responses, with 14 and 23F among those responsible for the most severe responses [33]. Nevertheless, we found no significant differences in mortality [34] as it has been reported that host factors have greater influence on outcomes [35].

This study has some limitations: 1) the CAP cases included were only those admitted to hospital and thus, the characteristics and distribution of serotypes in non-hospitalized patients is not known; 2) incidence rates could not be calculated and thus, the potential impact of childhood pneumococcal vaccination on pneumococcal CAP is not well characterized; 3 ) only 65 invasive samples were sent to the central microbiology laboratory and thus, invasive episodes may have been underestimated. Nevertheless, the results of this study offer some insights and suggestions as to the distribution of serotypes in pneumococcal CAP, and especially in non-invasive pneumococcal CAP.

In summary, we found that pneumococcal serotypes included in PCV13 cause a significant proportion of CAP in adults, but found no significant changes in cases due to PCV7 or PCV13 serotypes from 2011 to 2014, suggesting an insufficient indirect protection from pneumococcal vaccination of infants with PCV7/PCV13 during the study period. That proportion varied slightly according to the presence of underlying conditions and was higher in healthy adults, suggesting that a high percentage of adults would benefit from direct pneumococcal CAP protection. There is a need for strategies to implement pneumococcal conjugate vaccination to prevent pneumococcal pneumonia in adults in order to drastically reduce the current burden of the disease and to continue monitoring serotype distribution of non-invasive cases in the future.

\section{Acknowledgements}

Members of the CAPA study team are: A. Torres, C. Cilloniz, A. San José, F. Marco, E. Polverino, R. Amaro, (H. Clinic, Barcelona, Spain); R. Menéndez, R. Méndez, I. Amara, J.L. López Hontangas, B. Montull, A. Gimeno, A. Gil (H. Universitario y Politécnico La Fe, Valencia, Spain); PP. España, A. Uranga, A.P. Martínez de la Fuente, (H. Galdakao-Usansolo, Galdácano, Spain); E. Pérez-Trallero, J.M. Marimón, M. Ercibengoa (H. Universitario Donostia, San Sebastián, Spain); A. Fernández-Villar, M.I. Botana, F. Vasallo (H. Alvaro Cunqueiro, Vigo, Spain); C. Méndez, I. Cifuentes, C. Balseiro, A. García, M. Del Amo, J. Sáez and M.L. Samaniego (Pfizer S.L.U., Madrid, Spain).

\section{Funding}

This study was sponsored by Pfizer.

\section{Disclaimer}

The views expressed in this publication are those of the author(s) and not necessarily those of the sponsor.

\section{Competing interests}

R.M., A. T., P.P. E. and E. P-T report grants to their Institutions from Pfizer S.L.U., Madrid, Spain, for this study, and support from Pfizer S.L.U. for travelling to meetings for the study or other purposes during the conduction of the study.

C.M., I.C., C.B., A.G., J.S. and M.L. S. are employees of Pfizer S.L.U., Madrid, Spain.

\section{Ethics approval}

The ethics committee of each hospital approved the study. Patients provided written informed consent to participate in the study. 


\section{Appendix A. Supplementary material}

Supplementary data associated with this article can be found, in the online version, at http://dx.doi.org/10.1016/j.vaccine.2017.08. 007.

\section{References}

[1] Torres A, Blasi F, Dartois N, Akova M. Which individuals are at increased risk of pneumococcal disease and why? Impact of COPD, asthma, smoking, diabetes, and/or chronic heart disease on community-acquired pneumonia and invasive pneumococcal disease. Thorax 2015;70:984-9. http://dx.doi.org/10.1136/ thoraxinl-2015-206780.

[2] Feldman C, Anderson R. The role of Streptococcus pneumoniae in communityacquired pneumonia. Semin Respir Crit Care Med 2016;37:806-18. http://dx. doi.org/10.1055/s-0036-1592074.

[3] Waight PA, Andrews NJ, Ladhani SN, Sheppard CL, Slack MPE, Miller E. Effect of the 13-valent pneumococcal conjugate vaccine on invasive pneumococcal disease in England and Wales 4 years after its introduction: an observational cohort study. Lancet Infect Dis 2015;15:535-43. http://dx.doi.org/10.1016/ S1473-3099(15)70044-7.

[4] Chalmers JD, Campling J, Dicker A, Woodhead M, Madhava H. A systematic review of the burden of vaccine preventable pneumococcal disease in UK adults. BMC Pulm Med 2016;16:77. http://dx.doi.org/10.1186/s12890-0160242-0.

[5] Rodrigo C, Bewick T, Sheppard C, Greenwood S, Mckeever TM, Trotter CL, et al. Impact of infant 13-valent pneumococcal conjugate vaccine on serotypes in adult pneumonia. Eur Respir J 2015;45:1632-41. http://dx.doi.org/10.1183/ 09031936.00183614.

[6] Bonten MJM, Huijts SM, Bolkenbaas M, Webber C, Patterson S, Gault S, et al. Polysaccharide conjugate vaccine against pneumococcal pneumonia in adults. N Engl J Med 2015;372:1114-25. http://dx.doi.org/10.1056/NEIMoa1408544.

[7] Menendez R, Torres A, España P, Perez-Trallero E, Lopez-Hontagas JL, Marco F, et al. Differences in S. Pneumoniae serotypes distribution causing CAP in hospitalized adults in Spain according to comorbidities by a new urinary antigen detection (UAD) test. The CAPA study. Eur Respir J 2015; 46.

[8] Domenech A, Ardanuy C, Balsalobre L, Marti S, Calatayud L, De La Campa AG, et al. Pneumococci can persistently colonize adult patients with chronic respiratory disease. J Clin Microbiol 2012;50:4047-53. http://dx.doi.org/ 10.1128/JCM.02056-12.

[9] Quan TP, Fawcett NJ, Wrightson JM, Finney J, Wyllie D, Jeffery K, et al. Increasing burden of community-acquired pneumonia leading to hospitalisation, 1998-2014. Thorax 2016:thoraxjnl-2015-207688. doi: 10.1136/thoraxjnl-2015-207688.

[10] Pride MW, Huijts SM, Wu K, Souza V, Passador S, Tinder C, et al. Validation of an immunodiagnostic assay for detection of 13 Streptococcus pneumoniae serotype-specific polysaccharides in human urine. Clin Vaccine Immunol 2012;19:1131-41. http://dx.doi.org/10.1128/CVI.00064-12.

[11] Marimon JM, Ercibengoa M, Santacatterina E, Alonso M, Perez-Trallero E. Single-step multiplex PCR assay for determining 92 pneumococcal serotypes. J Clin Microbiol 2016;54:2197-200. http://dx.doi.org/10.1128/JCM.01156-16.

[12] Menendez R, Torres A, Aspa J, Capelastegui A, Prat C, Rodriguez de Castro F. Community acquired pneumonia. New guidelines of the Spanish Society of Chest Diseases and Thoracic Surgery (SEPAR). Arch Bronconeumol 2010;46:543-58.

[13] Halm EA, Teirstein AS. Clinical practice. Management of community-acquired pneumonia. N Engl J Med 2002;347:2039-45.

[14] Curcio D, Cané A, Isturiz R. Redefining risk categories for pneumococcal disease in adults: critical analysis of the evidence. Int J Infect Dis 2015;37:30-5. http: / dx.doi.org/10.1016/i.ijid.2015.05.003.

[15] Shea KM, Edelsberg J, Weycker D, Farkouh RA, Strutton DR, Pelton SI. Rates of pneumococcal disease in adults with chronic medical conditions. Open Forum Infect Dis 2014; 1: ofu024. doi: 10.1093/ofid/ofu024.

[16] Lujan M, Gallego M, Fontanals D, Mariscal D, Rello J. Prospective observational study of bacteremic pneumococcal pneumonia: Effect of discordant therapy on mortality. Crit Care Med 2004;32:625-31.

[17] Ardanuy C, Marimón JM, Calatayud L, Giménez M, Alonso M, Grau I, et al. Epidemiology of invasive pneumococcal disease in older people in Spain (2007-2009): Implications for future vaccination strategies. PLoS One 2012; 7. doi: 10.1371 /journal.pone.0043619.
[18] Moore MR, Link-Gelles R, Schaffner W, Lynfield R, Lexau C, Bennett NM, et al. Effect of use of 13-valent pneumococcal conjugate vaccine in children on invasive pneumococcal disease in children and adults in the USA: analysis of multisite, population-based surveillance. Lancet Infect Dis 2015;15:301-9. http://dx.doi.org/10.1016/S1473-3099(14)71081-3.

[19] Fenoll A, Granizo J-J, Gimenez M-J, Yuste J, Aguilar L. Secular trends (19902013 ) in serotypes and associated non-susceptibility of S. pneumoniae isolates causing invasive disease in the pre-/post-era of pneumococcal conjugate vaccines in Spanish regions without universal paediatric pneumococcal vaccination. Vaccine 2015; 33: 5691-9. doi: 10.1016/j.vaccine.2015.08.009.

[20] Bewick T, Sheppard C, Greenwood S, Slack M, Trotter C, George R, et al. Serotype prevalence in adults hospitalised with pneumococcal non-invasive community-acquired pneumonia. Thorax 2012;67:540-5.

[21] Sherwin RL, Gray S, Alexander R, McGovern PC, Graepel J, Pride MW, et al. Distribution of 13-valent pneumococcal conjugate vaccine Streptococcus pneumoniae serotypes in US adults aged $\geq 50$ years with communityacquired pneumonia. J Infect Dis 2013;208:1813-20. http://dx.doi.org/ 10.1093/infdis/jit506.

[22] Rodrigo C, Bewick T, Sheppard C, Greenwood S, Macgregor V, Trotter C, et al. Pneumococcal serotypes in adult non-invasive and invasive pneumonia in relation to child contact and child vaccination status. Thorax 2014;69:168-73. http://dx.doi.org/10.1136/thoraxinl-2013-203987.

[23] Gervaix A, Ansaldi F, Brito-Avo A, Azzari C, Knuf M, Martinon-Torres F, et al. Pneumococcal vaccination in Europe: schedule adherence. Clin Ther 2014;36 (802-12):e1. http://dx.doi.org/10.1016/i.clinthera.2014.03.001.

[24] Obando I, Munoz-Almagro C, Arroyo LA, Tarrago D, Sanchez-Tatay D, MorenoPerez D, et al. Pediatric parapneumonic empyema, Spain. Emerg Infect Dis 2008;14:1390-7. http://dx.doi.org/10.3201/eid1409.071094.

[25] Bender JM, Ampofo K, Korgenski K, Daly J, Pavia AT, Mason EO, et al. Pneumococcal necrotizing pneumonia in Utah: does serotype matter? Clin Infect Dis 2008;46:1346-52. http://dx.doi.org/10.1086/586747.

[26] van Hoek AJ, Sheppard CL, Andrews NJ, Waight PA, Slack MPE, Harrison TG, et al. Pneumococcal carriage in children and adults two years after introduction of the thirteen valent pneumococcal conjugate vaccine in England. Vaccine 2014;32:4349-55. http://dx.doi.org/10.1016/ i.vaccine.2014.03.017.

[27] Shiri T, Datta S, Madan J, Tsertsvadze A, Royle P, Keeling MJ, et al. Indirect effects of childhood pneumococcal conjugate vaccination on invasive pneumococcal disease: a systematic review and meta-analysis. Lancet Glob Heal 2017;5:e51-9. http://dx.doi.org/10.1016/S2214-109X(16)30306-0.

[28] Domenech A, Ardanuy C, Calatayud L, Santos S, Tubau F, Grau I, et al. Serotypes and genotypes of Streptococcus pneumoniae causing pneumonia and acute exacerbations in patients with chronic obstructive pulmonary disease. J Antimicrob Chemother 2011;66:487-93. http://dx.doi.org/10.1093/jac/ dkq480.

[29] Burgos J, Lujan M, Falcó V, Sánchez A, Puig M, Borrego A, et al. The spectrum of pneumococcal empyema in adults in the early 21st century. Clin Infect Dis 2011;53:254-61. http://dx.doi.org/10.1093/cid/cir354.

[30] Garcia-Vidal C, Ardanuy C, Tubau F, Viasus D, Dorca J, Liñares J, et al. Pneumococcal pneumonia presenting with septic shock: host- and pathogenrelated factors and outcomes. Thorax 2010;65:77-81. http://dx.doi.org/ 10.1136/thx.2009.123612.

[31] Burgos J, Luján M, Larrosa MN, Fontanals D, Bermudo G, Planes AM, et al. Risk factors for respiratory failure in pneumococcal pneumonia: the importance of pneumococcal serotypes. Eur Respir J 2014;43:545-53. http://dx.doi.org/ $10.1183 / 09031936.00050413$

[32] Sanchez CJ, Hinojosa CA, Shivshankar P, Hyams C, Camberlein E, Brown JS, et al. Changes in capsular serotype alter the surface exposure of pneumococcal adhesins and impact virulence. PLoS One 2011; 6. doi: 10.1371/journal. pone.0026587.

[33] Kalin M. Pneumococcal serotypes and their clinical relevance. Thorax 1998;53:159-62. http://dx.doi.org/10.1136/thx.53.3.159.

[34] Cillóniz C, Ewig S, Polverino E, Muñoz-Almagro C, Marco F, Gabarrús A, et al. Pulmonary complications of pneumococcal community-acquired pneumonia: incidence, predictors, and outcomes. Clin Microbiol Infect 2012;18:1134-42. http://dx.doi.org/10.1111/j.1469-0691.2011.03692.x.

[35] Naucler P, Darenberg J, Morfeldt E, Ortqvist A, Henriques Normark B. Contribution of host, bacterial factors and antibiotic treatment to mortality in adult patients with bacteraemic pneumococcal pneumonia. Thorax 2013;68:571-9. http://dx.doi.org/10.1136/thoraxjnl-2012-203106. 